ARTICLE

https://doi.org/10.1038/s41467-019-10058-7

\title{
Single-molecule kinetics of pore assembly by the membrane attack complex
}

\author{
Edward S. Parsons ${ }^{1}$, George J. Stanley ${ }^{1}$, Alice L.B. Pyne (10 1, Adrian W. Hodel ${ }^{1,2}$, Adrian P. Nievergelt (10 ${ }^{3}$, \\ Anaïs Menny ${ }^{4}$, Alexander R. Yon ${ }^{1,2}$, Ashlea Rowley ${ }^{5}$, Ralf P. Richter ${ }^{5,6,7}$, Georg E. Fantner (1) ${ }^{3}$, \\ Doryen Bubeck (iD ${ }^{4}$ \& Bart W. Hoogenboom (D) 1,2,8
}

The membrane attack complex (MAC) is a hetero-oligomeric protein assembly that kills pathogens by perforating their cell envelopes. The MAC is formed by sequential assembly of soluble complement proteins $\mathrm{C} 5 \mathrm{~b}, \mathrm{C} 6, \mathrm{C} 7, \mathrm{C} 8$ and $\mathrm{C} 9$, but little is known about the ratelimiting steps in this process. Here, we use rapid atomic force microscopy (AFM) imaging to show that MAC proteins oligomerize within the membrane, unlike structurally homologous bacterial pore-forming toxins. C5b-7 interacts with the lipid bilayer prior to recruiting $\mathrm{C} 8$. We discover that incorporation of the first $\mathrm{C} 9$ is the kinetic bottleneck of MAC formation, after which rapid C9 oligomerization completes the pore. This defines the kinetic basis for MAC assembly and provides insight into how human cells are protected from bystander damage by the cell surface receptor CD59, which is offered a maximum temporal window to halt the assembly at the point of $\mathrm{C} 9$ insertion.

\footnotetext{
${ }^{1}$ London Centre for Nanotechnology, University College London, London WC1H OAH, UK. ${ }^{2}$ Institute of Structural and Molecular Biology, University College London, London WC1E 6BT, UK. ${ }^{3}$ Laboratory for Bio- and Nano-Instrumentation, Swiss Federal Institute of Technology Lausanne (EPFL), 1015 Lausanne, Switzerland. ${ }^{4}$ Department of Life Sciences, Imperial College London, South Kensington Campus, London SW7 2AZ, UK. ${ }^{5}$ School of Biomedical Sciences, Faculty of Biological Sciences, University of Leeds, Leeds LS2 9JT, UK. ${ }^{6}$ School of Physics and Astronomy, Faculty of Mathematics and Physical Sciences, University of Leeds, Leeds LS2 9JT, UK. ${ }^{7}$ Astbury Centre for Structural Molecular Biology, University of Leeds, Leeds LS2 9JT, UK. ${ }^{8}$ Department of Physics and Astronomy, University College London, London WC1E 6BT, UK. Correspondence and requests for materials should be addressed to E.S.P. (email: e.parsons@ucl.ac.uk) or to B.W.H. (email: b.hoogenboom@ucl.ac.uk)
} 
$\mathrm{T}$ he formation of lethal membrane pores is a ubiquitous event in defence and attack between pathogens and their hosts $^{1-3}$. It plays a critical role in the lytic, antimicrobial activity of human serum, the discovery of which was an early milestone in immunology ${ }^{4}$, and decades of study have unravelled the interplay between proteins that lead to the formation of immune pores in microbial membranes. The formation of these membrane attack complex (MAC) pores represents the final step in the activation of the complement system, an integral component of innate immunity, which surveys our body for pathogenic bacteria and which 'complements' the ability of leukocytes to kill pathogens. Dysregulation of MAC formation has been implicated in human disease $\mathrm{e}^{5,6}$, and therapeutics that control complement are being harnessed for cancer immunotherapy ${ }^{7,8}$. Understanding how complement proteins assemble from innocuous soluble monomers into killer transmembrane pores can therefore contribute to developing strategies for treating human disease where the MAC is implicated ${ }^{5}$, and for repurposing the complement system as a potent immunotherapeutic ${ }^{9}$.

Assembly of the MAC is the end product of a complex series of biochemical interactions in which initially soluble complement proteins bind and undergo dramatic structural rearrangements to form a transmembrane pore. The resulting MAC pore is a heterooligomer formed from the irreversible, stepwise assembly of 7 different polypeptide chains: C5b, C6, C7, C8 (a hetero-trimer comprised of $\mathrm{C} 8 \alpha, \mathrm{C} 8 \beta$ and $\mathrm{C} 8 \gamma$ ) and $\mathrm{C} 9$, where 18 copies of $\mathrm{C} 9$ are required to complete the pore (Fig. 1a, inset, Supplementary Fig. 1). Triggered upon detection of a pathogen, activation of complement leads to the generation of $\mathrm{C} 5 \mathrm{~b}$ via the cleavage of $\mathrm{C} 5$ by membrane-bound $\mathrm{C} 5$-convertase enzymes ${ }^{10}$. C5b is a metastable intermediate that rapidly sequesters $\mathrm{C}^{11}$. Recruitment of $\mathrm{C} 7$ unfurls a lipophilic domain upon binding, while integration of C8 into the assembly is accompanied by an initial insertion into the membrane. The C5b-8 initiator complex then binds C9 and undergoes unidirectional, clockwise oligomerization (with 18 copies of C9) to complete an $11 \mathrm{~nm}$ wide transmembrane pore, as characterised in increasing structural detail by cryo-electron microscopy (cryoEM) $)^{12-16}$. Together with crystallographic structures of component proteins, high-resolution cryoEM analyses of the full pore have identified regulatory roles for auxiliary domains ${ }^{15,17,18}$ that control the transition from stable proteins in our blood to lethal transmembrane pores. However, it remains unclear which are the rate-limiting steps in the assembly pathway of these complement proteins.

As there is no known lipid or receptor specificity for MAC membrane insertion, the kinetics of pore assembly drives both the rapid innate immune response to pathogens and dictates how the MAC can be most effectively inhibited on membranes of selfcells. For human cells, the only known membrane-associated inhibitor of MAC assembly is CD59, a glycosylphosphatidylinositol (GPI) anchored cell surface receptor. CD59 binds the transmembrane residues of $\mathrm{C} 8$ and $\mathrm{C} 9^{19}$, preventing pore formation and further oligomerization of $\mathrm{C}^{20}$. The kinetics of MAC formation must allow a temporal window such that inhibitory factors can interfere at appropriate stages in the assembly pathway. Therefore, a kinetic analysis of MAC assembly will provide a much-needed framework to understand how CD59 inhibits lysis.

To understand the molecular mechanism and kinetics underpinning how and when the MAC assembly becomes cytolytic, we sought to track the progression of the complement terminal pathway at the level of single pores. Using rapid atomic force microscopy (AFM) imaging on supported model membranes, we visualize the initial interactions of complement proteins with the membrane, and resolve the kinetics of MAC pore formation. Together these data reveal the overall rate of the assembly process and identify which steps in the pathway are rate-limiting.

\section{Results}

MAC forms pores in bacterial model membranes. To enable AFM tracking of MAC self-assembly at single-molecule resolution, we developed a model membrane system that supported the formation of transmembrane pores. We sequentially incubated complement proteins C5b6, C7, C8 and C9 at physiological concentrations $^{10,21}$ on supported bilayers formed from E. coli lipid extract ${ }^{22}$ and from pseudo E. coli lipid mixtures comprised of 1,2-dioleoyl-sn-glycero-3-phosphocholine (DOPC), 1,2-dioleoyl-sn-glycero-3-phospho-(1'-rac-glycerol) (DOPG) and 1,2dioleoyl-sn-glycero-3-phosphoethanolamine (DOPE). In our model comprised of synthetic lipids, we sought to harness the physico-chemical properties of the bacterial membrane: PG lipids

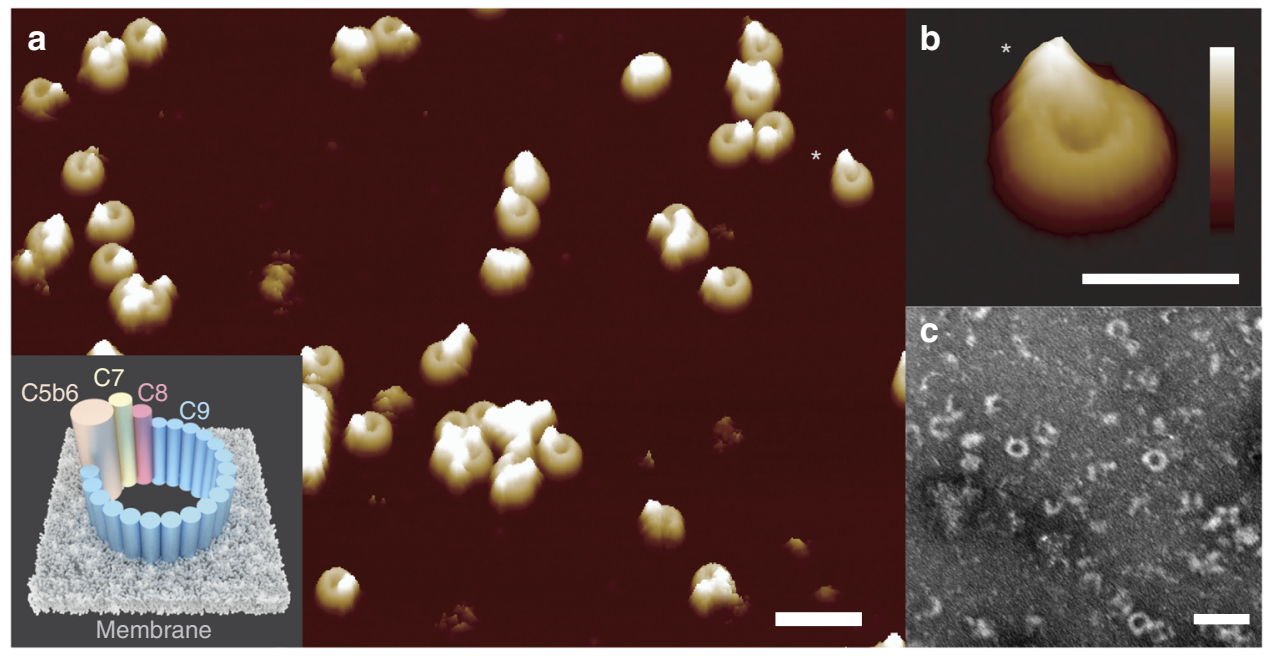

Fig. 1 Formation of MAC on bacterial model membranes. a 3D AFM representation of the endpoint MAC pore structure on supported bilayers composed of an E. coli lipid extract. Inset: Schematic of the MAC, self-assembled from complement proteins C5b6, C7, C8 and C9, embedded within a lipid membrane. b Zoom-in of a single MAC pore (marked with an asterisk in a). c Negative-stain EM of E. coli lipid bilayers deposited on silicon dioxide grids, sequentially incubated with complement proteins C5b6, C7, C8 and C9, resulting in characteristic MAC rings observed in the bilayer membrane. Scale bars: a, c 50 nm, b $25 \mathrm{~nm}$. Height scale (scale inset in b), a, b $20 \mathrm{~nm}$ 
harbour negative charge in their phosphoglycerol headgroup, whilst PE introduces a degree of stored curvature elastic stress into the plane of the membrane ${ }^{23}$. Inspection by AFM and fluorescence recovery after photobleaching (FRAP) reveals a single continuous supported bilayer with rapid in-plane diffusion (Supplementary Fig. 2).

High-resolution AFM images of the resulting end-point MAC pores are consistent with cryo-EM reconstructions (Supplementary Fig. 3a-d). We clearly resolve the lumen of the $\beta$-barrel pore in addition to the protruding C5b stalk that hallmarks the MAC (Fig. 1, Supplementary Fig. 1) ${ }^{12-16}$. Vesicle lysis assays corroborate the functional requirement of both a C5b-8 'initiator' and $\mathrm{C} 9$ 'propagators' for MAC to form lytic pores (Supplementary Fig. 3e); and negative-stain EM on lipid bilayers shows that C5b-8 is required for the oligomerization and insertion of $\mathrm{C} 9$ within the membrane (Supplementary Fig. 4). Together, these data confirm that the C5b-8 initiator complex, comprised of C5b6 in complex with $\mathrm{C} 7$ and $\mathrm{C} 8$, is essential for the formation of a functional MAC within the bacterial model membrane. Finally, we verified that an equivalent degree of MAC formation was observed across the lipid compositions used in this work (Supplementary Fig. 5).

C5b-7 initiates MAC assembly on the target membrane. Previous biochemical studies of MAC formation were performed on an ensemble of erythrocyte and liposome membranes, preventing analysis of individual pores ${ }^{24-26}$. By contrast, our experimental system facilitates a stepwise study of MAC assembly at the singlemolecule level. Upon addition of C5b6 to a bacterial model membrane, we observe features of few-nanometre dimensions (Fig. 2a, Supplementary Fig. 6). Their transient nature on the membrane and protruding structure pose a challenge for AFM imaging 27,28 , in practice putting them at the detection limit of AFM. At higher sampling rates (Supplementary Movie 1), AFM force sensitivity was sacrificed for enhanced speed of imaging - in these instances, early assemblies are more difficult to detect. Further in situ incubation with C7 yields an increase in the number of such features, which appear with enhanced clarity after the addition of C8 (Fig. 2a, Supplementary Fig. 6). These data indicate that C5b6 interacts with the bacterial model membrane and becomes more resistant to the movement of the scanning AFM tip following assembly with C7 and C8.

To rule out artefacts due to the inherent invasiveness of the AFM measurement, we also performed binding assays by quartz crystal microbalance with dissipation monitoring (QCM-D) to study the protein assembly on/in membranes ${ }^{29-32}$, which correlate with our AFM data. Briefly, we prepared supported lipid bilayers on silicon dioxide coated QCM-D sensors, with which we could detect membrane binding of complement proteins $^{31,32}$ (Fig. 2b, Supplementary Fig 7). Upon addition of C5b6, we observe shifts in frequency $(\Delta f)$ and dissipation $(\Delta D)$ with large variability between samples. Incubations with $\mathrm{C} 5 \mathrm{~b}-7$ followed by $\mathrm{C} 8$ show consistently strong responses as stable complexes bind to the membrane and could not be removed by washing with excess buffer. From the analysis of the binding kinetics, and the C5b-7 dose-dependent C8 and C9 binding response, we show that the stoichiometry of the complex is determined by C5b-7 binding (Supplementary Fig. 8a). The QCM-D data also reveal that the protein complex becomes mechanically less compliant by its insertion into the membrane upon addition of C8 and C9 (Supplementary Fig. 8b) ${ }^{33}$. Upon addition of $\mathrm{C}$, we observe a final shift in frequency, corresponding to the inserted MAC pore. Taken together, these results demonstrate that the MAC is initiated by C5b6, which is explicitly required for $\mathrm{C} 7$ to bind to the membrane and propagate pore formation.

C9 monomers are recruited directly from solution. MAC proteins are structurally homologous to the immune protein perforin and to bacterial cholesterol dependent cytolysins, both of which form pores through the homo-oligomerization of membraneassociated monomers $3,27-30,34-37$. We next explored whether the hetero-oligomeric MAC pore could assemble via analogous membrane-bound C9 oligomers, prior to its association with C5b-8. However, from the absence of C9 on E. coli lipid bilayers in the electron microscopy and AFM images in Supplementary Fig. 5, we conclude that such intermediates, if existing at all, are only transiently bound to the membrane. Upon addition of C9, AFM imaging (Supplementary Movie 1) does not show the characteristic pre-pore carpet that was detected by AFM for
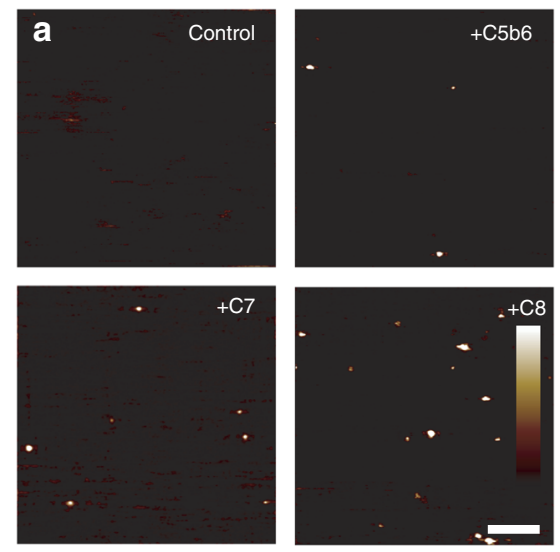

b

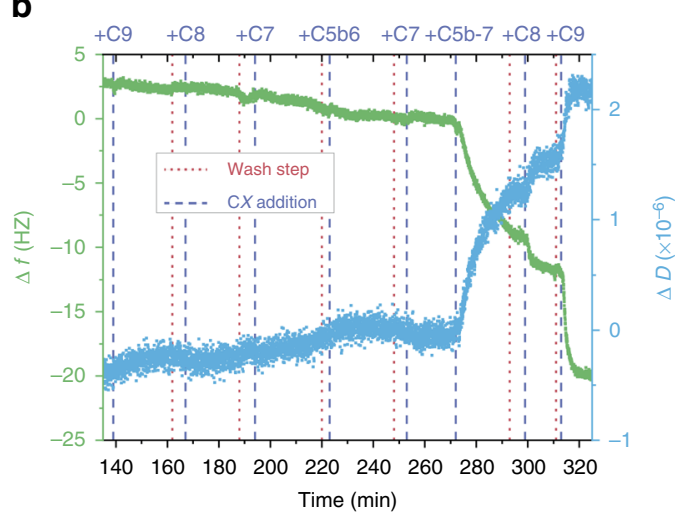

Fig. 2 C5b-7 initiates MAC formation and recruits C8 and C9 directly from solution. Binding of complement components C5b6, C7 and C8 to supported

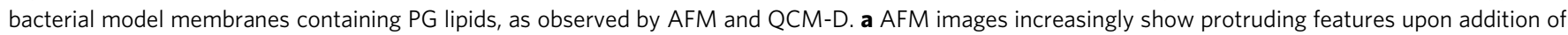
C5b6, C7 and C8 to a bilayer formed of DOPE:DOPG (50:50 mol\%). Scale bar: $200 \mathrm{~nm}$, height scale: $1 \mathrm{~nm}$. b QCM-D binding assay following the addition of complement proteins, in reverse sequence followed by the forward sequence, to a lipid bilayer formed of DOPC:DOPE:DOPG (47.5:47.5:5 mol\%) on a silicon dioxide coated QCM-D sensor. In isolation, complement proteins C7, C8 and C9 do not bind to the membrane, requiring C5b6 to initiate the interaction. Distinct steps in both frequency $(\Delta f)$ and dissipation $(\Delta D)$ upon sequential addition of $C 5 \mathrm{~b}-7$, C8 and $C 9$ demonstrate protein binding to the membrane; wash steps (denoted by dotted red lines) between additions do not lead to a reduction in the signal size, revealing that the protein binding is stable. The dissipation shift increases upon binding, reflecting the relative softness of the protein complexes in the lipid bilayer film 

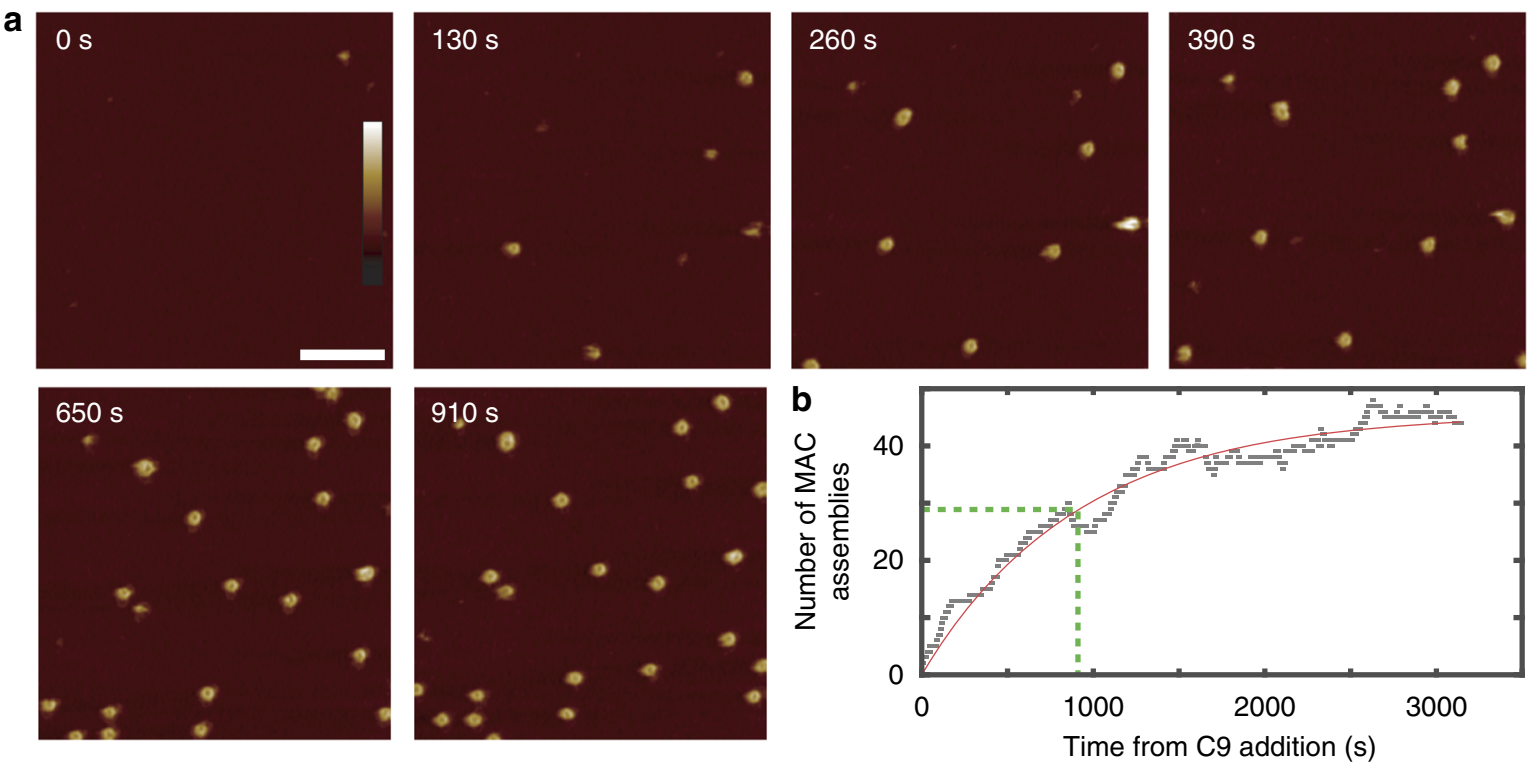

Fig. 3 AFM shows that initial insertion of C9 is rate limiting in MAC assembly. a Time-lapse AFM imaging of MAC assembly on supported bilayers formed of E. coli lipid extract (see Supplementary Movie 1 for full data set). MAC assembly events initiate at distinct time points, with each MAC pore rapidly completing within a few frames; this implies a rate-limiting-step corresponding to initial insertion of C9. Scale bar: $200 \mathrm{~nm}$; height scale (colour scale bar inset in image for $t=0 \mathrm{~min}$ ): $40 \mathrm{~nm}$. b Number of MAC assemblies detected (see Methods) with time, indicated as grey squares; the solid red line represents fitting with the function $A\left(1-\exp \left(-t / \tau_{\text {init }}\right)\right)$, where $\tau_{\text {init }}=912 \pm 32 \mathrm{~s}$ represents the characteristic time of MAC pore appearance, highlighted by the dashed green lines. The completion time of each individual MAC is much shorter than $\tau_{\text {init }}$

perforin $^{27}$ and the cholesterol dependent cytolysin suilysin ${ }^{28}$. Instead, we observe a planar membrane background that remains featureless until MAC pores emerge, implying the absence of any pre-pore oligomers until the pore is assembled within the membrane. The static nature of these MACs confirms their membrane-inserted, pore character ${ }^{27}$. To further validate that $\mathrm{C} 9$ binds directly to C5b-8 and does not form C9 pre-pores on the membrane, we performed QCM-D analysis of bilayers that were incubated with complement proteins in the reverse order (i.e. C9, C8, C7, C5b6, Fig. 2b), with wash steps in between. C9, C8 and C7 separately did not show any significant binding to the membrane in the absence of other proteins. Taken together, these data demonstrate that $\mathrm{C} 9$ monomers are recruited directly from solution to the nascent pore and not via a membrane-bound C9 intermediate.

Initial C9 insertion is a kinetic bottleneck in MAC assembly. By tracking the appearance and evolution of individual pores, we next determined the reaction kinetics that govern MAC assembly. Upon association with C5b-8, C9 oligomerizes to complete a transmembrane pore. With a frame rate of $6.5 \mathrm{~s}$ per frame, we used AFM to visualise C9 oligomerization in real time at $30^{\circ} \mathrm{C}$ (Fig. 3a, Supplementary Movie 1, in which $t=0$ is defined as the time of C9 addition); data recorded at the physiological $37^{\circ} \mathrm{C}$ showed similar kinetics (Supplementary Fig. 9, Supplementary Movie 2). A single MAC pore is formed within the first few frames, immediately after addition of C9. The pore persists in isolation for $30 \mathrm{~s}$, after which multiple (3-5) pores appear simultaneously, and the number of pores augments at aradually decreasing rate, up to 50 MAC pores in the field of view at the end of the recording (Supplementary Movie 1 and Fig. 3).

Remarkably, complete MAC pores continue to appear over an hour; yet the completion of each individual MAC occurs at the timescale of seconds to minutes (Fig. 3a, Supplementary Movie 1). Hence, two distinct kinetic steps are observed upon C9 addition to the nascent MAC. Firstly, we observe slow C9 binding to C5b8 , which is taken as our initial detection of a pore-forming event after the addition of C9. We define kinetics of this event by a characteristic initiation time $\tau_{\text {init }}$. Secondly, our data show rapid C9 oligomerization to a $\mathrm{C} 5 \mathrm{~b}-8 \mathrm{C} 9_{n}$ MAC pore. We define the time for each growing pore to fully assemble as $\tau_{\text {olig. }}$. Consistent with the description of these two distinct kinetic steps, the vast majority of end-point MACs in our data are complete, ringshaped pores. If the rate of initial C9 insertion were faster than the subsequent oligomerization reaction, kinetically trapped, arcshaped assemblies would occur due to monomer depletion, as has been observed for other pore-forming proteins ${ }^{27,28,34}$. To determine whether the kinetic bottleneck is attributed to C8 incorporation or insertion of the first $\mathrm{C} 9$, we allowed an extended temporal window after the addition of $\mathrm{C} 8$ and prior to adding C9: we confirm by time-lapse AFM imaging that this does not lead to more efficient/faster MAC formation (Supplementary Fig. 10, Supplementary Movie 3).

Rapid AFM imaging allows quantification of reaction times. To quantify the reaction time of initiation and oligomerization, we analysed rapid AFM imaging data of pore assembly. Considering the up to 100-fold difference between the timescales of MAC appearance (initiation) and completion (C9 oligomerisation), the timescale of completion can be assumed negligible relative to that of initiation. We detected pores by cross-correlation during particle tracking (see methods), and take pore appearance as a proxy that reports on the time of initiation of MAC assembly after addition of $\mathrm{C} 9$ to the reservoir. In doing so, we determine a characteristic initiation time $\tau_{\text {init }}=912 \pm 32 \mathrm{~s}$ (Fig. 3b).

The kinetics of C9 oligomerization were determined by tracking areas with individual pores from just before the point of initial detection to after completion (Fig. 4, Supplementary Movie 4). Image sequences of these tracks show distinct intermediates of a growing pore as the MAC completes (Fig. 4a, Supplementary Fig. 11). To quantify the timescale of the transition we use the average frame height (defined by the average pixel intensity) as a proxy to report on the completeness of the pore. As the pore evolves we observe a gradual increase in 

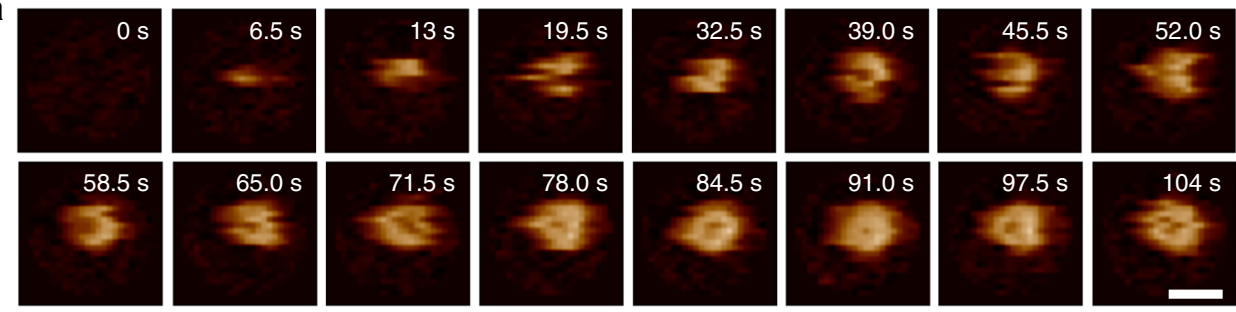

b

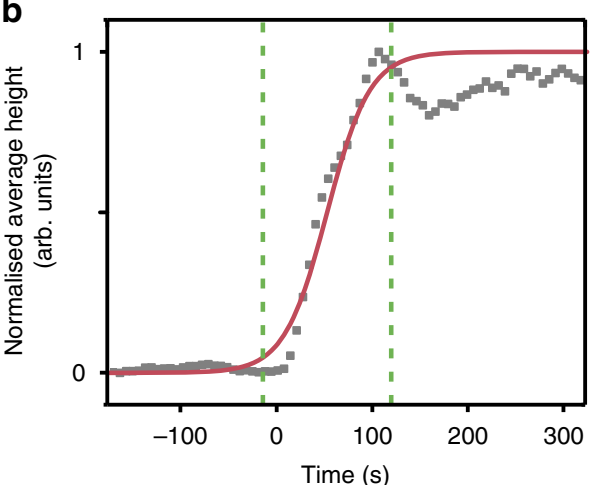

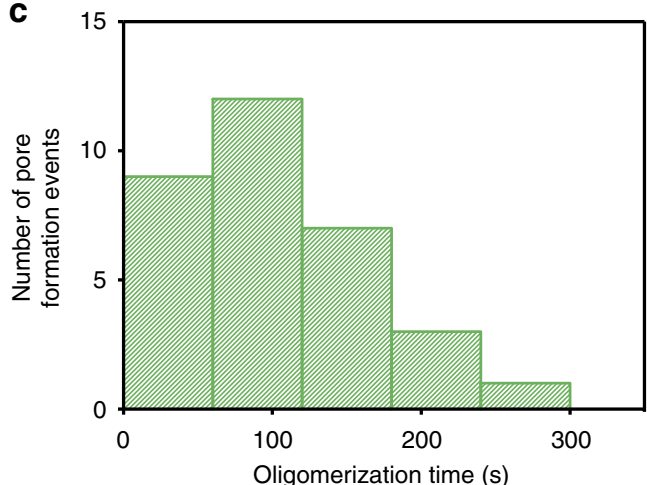

Fig. 4 Real-time imaging of C9 oligomerization. a AFM image sequence of MAC assembly, cropped from data shown in Fig. 3 and Supplementary Movie 1. C9 oligomerization completes within frames shown (104 s, $6.5 \mathrm{~s} /$ frame; $0 \mathrm{~s}$ is here approximately defined by the frame preceding detection of the growing MAC). Scale bar: $30 \mathrm{~nm}$, height scale (see colour scale bar in Fig. 1): $16 \mathrm{~nm}$. b The normalised average frame height versus time for a single pore forming event (corresponding to event shown in $\mathbf{a}$ ), plotted here as a measure for completion of MAC assembly. The red line represents a sigmoidal fit to the data, as a generic and mathematically convenient description of a smooth transition between pore absence and pore completion. The C 9 oligomerization time is determined from the width of the transition, highlighted by green dashed lines (see Supplementary Fig. 12 for details). c Distribution of oligomerization times, extracted from $n=33$ isolated pore forming events in 6 independent experiments

the average height in the frame, which plateaus as the MAC reaches a final state (Fig. 4b, Supplementary Figs. 11-12, Supplementary Movie 4). Previous structural studies defined the stoichiometry of the complete MAC as having 18 copies of $\mathrm{C}^{12}$. To measure the time required to add 17 copies of $\mathrm{C} 9$ after the initiation event, $\tau_{\text {olig, }}$, we record the width of the transition between detection of the initial event and appearance of a complete MAC pore, $\tau_{\text {olig }}=112 \pm 17 \mathrm{~s}$ (Fig. 4c). This is an order of magnitude shorter than $\tau_{\text {init }}$, and implies that the average time per addition of each of the remaining 17 C9 subunits $\left(\tau_{+}=\tau_{\text {olig }} /\right.$ $17=6.6 \pm 1.0 \mathrm{~s})$ is more than two orders of magnitude shorter than $\tau_{\text {init }}$.

A kinetic model for $\mathrm{C} 9$ assembly in the MAC. Given that $\tau_{\text {init }} \gg \tau_{+}$, we can interpret our data in terms of the separate reactions $\mathrm{C} 5 \mathrm{~b}-8+\mathrm{C} 9 \stackrel{k_{\text {init }}}{\rightarrow} \mathrm{C} 5 \mathrm{~b}-8(\mathrm{C} 9)_{1}$ and $\mathrm{C} 5 \mathrm{~b}-8(\mathrm{C} 9)_{n}+$ $\mathrm{C} 9 \stackrel{k_{+}}{\rightarrow} \mathrm{C} 5 \mathrm{~b}-8(\mathrm{C} 9)_{n+1}(1 \leq n<18)$, with respective rate constants $k_{\text {init }}$ and $k_{+}$. We observe that the C9 oligomerization times are independent of the time at which the reaction is initiated (Supplementary Fig. 13), consistent with C9 being in excess. As such, the interpretation of this reaction scheme can be further simplified by the assumption of an excess and thus approximately constant C9 concentration in solution $([\mathrm{C} 9] \approx 1.4 \mu \mathrm{M}$ ) over the duration of our experiments. Consequently, under our experimental conditions the final number of MAC pores is set by the number of C5b- 8 complexes on the membrane. These assumptions lead to the exponential time dependence of MAC appearance as observed in Fig. 3b, with $k_{\text {init }} \approx \tau_{\text {init }}{ }^{-1}[\mathrm{C} 9]^{-1}=0.78 \mathrm{~s}^{-1} \mathrm{mM}^{-1}$ (see Methods). In addition, assuming a constant rate of $\mathrm{C} 9$ addition after the insertion of the first C9, we find $k_{+} \approx \tau_{+}{ }^{-1}[\mathrm{C} 9]^{-1}=108 \mathrm{~s}^{-1} \mathrm{mM}^{-1}$ (see Methods). This more quantitative analysis confirms that the initial insertion of $\mathrm{C}$, together with its binding to $\mathrm{C} 5 \mathrm{~b}-8$, is the major rate-limiting step in MAC assembly. Interestingly, this rate-limiting step coincides with the stage where MAC pore formation is inhibited by CD59, which is present on the surface of human cells to prevent them from being lysed by complement ${ }^{19,20,38}$.

\section{Discussion}

The MAC represents a biomedically important system in which to probe how unique individual proteins self-assemble into a macromolecular functional unit. Once initiated, five soluble complement proteins sequentially and irreversibly self-assemble into a hetero-oligomeric pore that opens up an $11 \mathrm{~nm}$ hole in a fluid lipid bilayer. While there is an extensive (and still expanding) body of structural and functional information documenting the MAC and its constituent components, the pathway and kinetics of its assembly have been more difficult to study ${ }^{24}$. Here we have presented rapid AFM imaging data that track MAC assembly at the single-pore level in real-time. Furthermore, we have derived kinetic models for initiation and oligomerization of C9 that explain how rate-limiting assembly intermediates can be captured by our body's self-defence mechanism to prevent disease.

Based on the results presented here, we define a kinetic pathway of MAC assembly (Fig. 5). C5b6/C5b-7 binds to bacterial lipids and serves as a platform for coordinating the sequential assembly of the MAC at the target membrane, recruiting C8. Downstream, the C5b-8 initiator complex is explicitly required for membrane insertion of the initial C9 molecules. Our data reveal that this initiation phase, which relates to the insertion of the first $\mathrm{C} 9$, is the rate-limiting step. A rapid oligomerization phase completes the transmembrane MAC pore, as further copies of C9 bind and insert into the membrane directly from solution. Specifically, the binding of the initial C9 to C5b-8 is characterised by a rate constant that is more than two orders of magnitude 


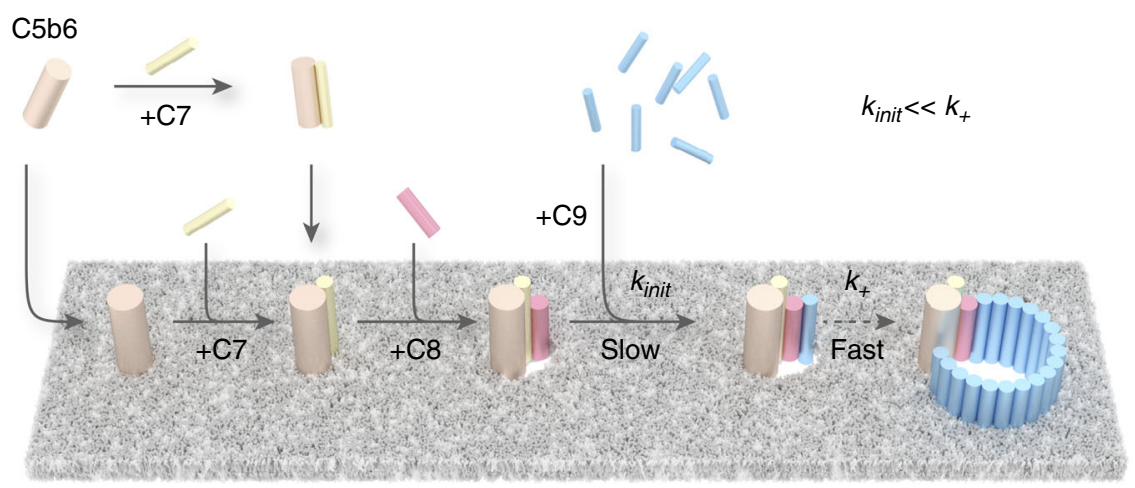

Fig. 5 Schematic of MAC assembly. Upon formation, C5b6 templates the assembly, recruiting C7 and C8 to the nascent MAC. The C5b-7 complex is irreversibly bound to the membrane. Insertion of the first C9 is the rate limiting step in the MAC assembly; once this barrier has been overcome, subsequent C9 binding/insertion occurs more than two orders of magnitude faster than the initial C9 insertion

smaller than that for the subsequent binding of $\mathrm{C} 9$ to $\mathrm{C} 5 \mathrm{~b}-8 \mathrm{C} 9_{\mathrm{n}}$ $(1 \leq n<18)$, and occurs more slowly than C5b-8 formation.

Since the propagation of $\mathrm{C} 9$ oligomerization is so much faster than the initiation, we predict that there will be a low probability of incomplete, arc-shaped assemblies that are kinetically trapped due to monomer depletion. In agreement with this prediction, we find that MAC assembly favours formation of complete, ringshaped pores (Fig. 1a), in contrast to kinetically trapped arc pores observed for other pore-forming protein $\mathrm{s}^{27,28,34}$. In summary, we show that once activated, the MAC pore rapidly assembles into complete ring lesions in the target membrane.

By having distinct initiation and propagation stages, C9 assembly in the MAC resembles pore formation of the related immune protein perforin, where the rate limiting step is the insertion of a small (membrane-bound but not yet membraneinserted) pre-pore assembly, acting as a nucleation site from which to grow a transmembrane pore ${ }^{27,39}$. Although we do not observe any membrane-bound C9 pre-pore assemblies, the C5b-8 initiator complex, together with a C9 molecule yet to undergo its transmembrane transition, could serve a similar function. Our data provides evidence that MAC is a growing pore, similar to perforin. Distinct initiation and propagation stages have also been proposed for bacterial cholesterol-dependent cytolysins (CDCs), with the formation of a stable membrane-bound dimer followed by the addition of further monomers ${ }^{35}$. However, the CDCs differ from the MAC and perforin in that they undergo a concerted oligomeric pre-pore-to-pore transition, after which no further assembly events have been observed ${ }^{3,28,34}$. In a bacterial membrane that harbours a dense proteinaceous network of porins ${ }^{10,40}$, such a collective pre-pore-to-pore transition is likely to face a large free energy barrier; instead, a growing pore that directly recruits individual monomers from solution can act as a jack that prises open a hole within the porin lattice.

The transition from soluble monomers to a transmembrane $\beta$ barrel pore requires dramatic conformational changes in the pore-forming domain of complement proteins. The membrane attack complex-perforin/cholesterol dependent cytolysin (MACPF/CDC) domain is comprised of a central kinked $\beta$-sheet with two helical sub-regions (TMH; transmembrane hairpins) that unfurl to form membrane-inserted $\beta$-hairpins. This transition is accompanied by unbending of the central $\beta$-sheet and the displacement of a latch that releases the TMH bundles. For CDCs, this latch is manifested as a fifth $\beta$-strand of the central sheet that upon pore-formation converts to a helix-turn-helix, enabling oligomerization $^{36,37}$. For MACPF-containing complement proteins, the latch remains helical and moves as a unit to release transmembrane residues ${ }^{13,15}$. Interestingly, the rate-limiting step of MAC assembly, as identified here, coincides with unfurling of the hairpins of the first C9 into the membrane. This can be related to the recent structural insight that $\mathrm{C} 9$ can bind to the C5b-8 initiator complex before inserting into the membrane; however, to propagate oligomerization, it requires conformational changes with the MACPF domain that accompany membrane insertion $^{15}$.

Our results show that C5b-7 initiates MAC assembly at the membrane with subsequent protein components C8 and C9 integrated directly from solution. Upstream, the C5b6 crystal structure demonstrated that prior to interacting with membrane lipids, C6 membrane-interacting residues remain in their soluble helical form ${ }^{17}$; however, the C5b6 complex has been shown to associate with lipid bilayers ${ }^{13}$. The dual behaviour of C5b6 is reflected in the variability in our results (Fig. 2b, Supplementary Fig. 7), suggesting that interactions are mediated by a combination membrane defects and electrostatics ${ }^{13}$. It is conceivable that C5b6 membrane-binding is dominated by electrostatic interactions of negatively charged lipid headgroups (such as PG lipids commonly found in Gram-negative bacteria ${ }^{41}$ ) with either the thrombospondin (TS) 1 domain of $\mathrm{C}^{42}$ or by its unfurled membrane interacting $\beta$-hairpin ${ }^{13}$, both of which expose an interface rich in positive charge. Such lipid headgroup dependence also emerges from studies on MAC binding ${ }^{31,32}$ and complement activation on model membranes ${ }^{43}$. The observed membrane binding of C5b6 is consistent with recent work on bacteria $^{10}$, reporting that the downstream efficiency of MAC formation is greatly enhanced when C5b6 is actively formed by C5 convertases bound to the bacterial surface. Our results here provide the rationale for this observation. We propose that nascent C5b6 can, under certain conditions, interact with the bacterial membrane upon its generation by the $\mathrm{C} 5$ convertase. C7 greatly enhances the membrane-bound stability of the nascent complex as it unfurls a lipophilic domain, and recruits $\mathrm{C} 8$ directly to the target membrane. This facilitates binding and oligomerization of $\mathrm{C} 9$, thus generating the functional pores that kill the bacterium.

Although the here discussed lipid dependence suggest some MAC specificity for bacterial targets, C5b-8-initiator complexes can deposit and progress to cytolytic pores on host cells if not properly controlled. Therefore, human cells express CD59 on their surface, which disrupt MAC assembly from the point of C5b-8 formation onwards ${ }^{19,20,38,44,45}$. Specifically, CD59 interacts with MAC precursors at two distinct stages. Firstly, CD59 can bind after incorporation of $\mathrm{C} 8(\mathrm{C} 5 \mathrm{~b}-8)$ and prevent bilayer perforation by the C8a chain ${ }^{44}$. Secondly, CD59 can bind after addition of C9 (C5b-9) to inhibit further oligomerization and pore formation ${ }^{44}$. CD59 inhibits MAC assembly by binding to the TMH $\beta$-hairpin on the leading-face of C8a (residues 334-385) ${ }^{45}$ 
and to a buried 6 amino acid sequence of C9 (residues 366-371) that is exposed upon binding C $5 \mathrm{~b}-8^{38}$. However, the structural basis for how CD59 inhibits complement proteins is unclear. It is not known whether C9 initiates insertion of its hairpin into the membrane prior to binding CD59 or if CD59 binds C8 and C9 simultaneously.

Our data highlight a rate-limiting step at the association of C9 with the C5b-8 MAC precursor. This allows a maximum temporal window for the mechanism by which human cells are protected from autoimmune attack by the MAC: our model favours a mechanism whereby $\mathrm{C} 9$ has not unfurled into the membrane immediately upon binding C5b-8, allowing CD59 to inhibit MAC formation. The here reported initiation step would thus correspond to the insertion of the first C9 into the membrane, facilitating fast recruitment of additional C9 to the growing transmembrane complex. When CD59-mediated inhibition is overcome by antibody-based drugs such as rituximab that facilitate MAC-induced killing of chronic lymphocytic leukaemia Bcells, cell death follows at the $\sim 100 \mathrm{~s}$ time scale 7 . This is consistent with the oligomerization kinetics of a single pore observed here in vitro, suggesting that only few pores are sufficient to lyse a Bcell.

In summary, we have determined the pathways and kinetics of assembly for a hetero-oligomeric protein complex by molecularscale measurements. These assembly kinetics govern how MAC kills bacteria and how our body's self-defence mechanism prevents membrane damage, which may also be relevant for complement dependent cytotoxicity in cancer immunotherapy ${ }^{7}$. Finally, we anticipate that our findings will guide the interpretation of ongoing studies towards an atomistic model of MAC structure.

\section{Methods}

Materials. E. coli lipid extract (total), 1,2-dioleoyl-sn-glycero-3-phosphocholine (DOPC), 1,2-dioleoyl-sn-glycero-3-phosphoethanolamine (DOPE), 1,2-dioleoylsn-glycero-3-phospho-(1'-rac-glycerol) (DOPG) and 1-palmitoyl-2-(dipyrrometheneboron difluoride)undecanoyl-sn-glycero-3-phosphocholine (TopFluor ${ }^{\circledast}$ PC) were purchased from Avanti Polar Lipids (Alabama, USA) as a powder and stored at $-20^{\circ} \mathrm{C}$ prior to use. Complement proteins C5b6, C7, C8 and C9 were purchased as proteins purified from human serum from CompTech (Texas, USA) and stored at $-80{ }^{\circ} \mathrm{C}$ prior to use. For the rapid AFM imaging experiments, lysis assays, negative-stain EM and FRAP experiments shown here, E.coli lipid extract was used. For AFM imaging of intermediates, an equimolar mixture of DOPG: DOPE was used. For QCM-D binding assays, a lipid mixture of DOPC:DOPE: DOPG (47.5:47.5:5 mol\%) was used, as formation of continuous bilayers containing high molar ratios of charged lipid ( $>30 \mathrm{~mol} \%$ ) were not attainable on the silicon dioxide QCM-D sensors used here ${ }^{46}$.

Preparation of lipid vesicles. Pure lipids were dissolved in chloroform at $10 \mathrm{mg} / \mathrm{mL}$ and mixed in solution to give a lipid mixture at a desired molar ratio. The lipid-inchloroform solution was then dried in a glass vial under a stream of nitrogen gas to give $1 \mathrm{mg}$ of lipid as a thin film. The lipid film was hydrated in buffer $(20 \mathrm{mM}$ HEPES, $120 \mathrm{mM} \mathrm{NaCl}, \mathrm{pH}$ 7.4), vortexed and bath sonicated to give a cloudy lipid suspension. The suspension was then passed through a $50 \mathrm{~nm}$ polycarbonate membrane (GE Healthcare Lifesciences) 15 times to yield a clear suspension of small unilamellar vesicles (SUVs). All lipid species used had a gel-to-fluid transition below room temperature, and therefore were assumed to be miscible without heating.

AFM sample preparation. Supported lipid bilayers were formed by injecting 4.5 $\mu \mathrm{L}$ of the SUV suspension to a freshly cleaved mica disk (6 mm diameter) under 18 $\mu \mathrm{L}$ of incubation buffer (20 mM HEPES, $120 \mathrm{mM} \mathrm{NaCl}, \mathrm{pH} 7.4) . \mathrm{CaCl}_{2}$ solution $(2.5 \mu \mathrm{L}, 100 \mathrm{mM}$ in incubation buffer) was added to give a final calcium concentration of $10 \mathrm{mM}$; this induces the rupture of the vesicles onto the mica support over an incubation period of approximately $30 \mathrm{~min}$. Excess vesicles were then removed from the supernatant by rinsing with $500 \mu \mathrm{L}$ of incubation buffer, to yield a uniform bilayer free of adsorbed vesicles (as assessed by AFM imaging). All SUVs were incubated at room temperature, above their gel-to-fluid transition temperature.

Endpoint MAC pores were formed by incubating the supported bilayer in a humid chamber at $37^{\circ} \mathrm{C}$ and sequentially adding complement proteins $\mathrm{C} 5 \mathrm{~b} 6, \mathrm{C} 7$, C8 and C9 at 5 min intervals, with a final $15 \mathrm{~min}$ incubation after the addition of C9 prior to initiating the AFM experiment. Final concentrations of complement proteins were $\sim 80 \mathrm{nM}$ for C5b6, C7 and C8 and $1.4 \mu \mathrm{M}$ for C9. Excess soluble protein was removed prior to imaging by washing with $5 \times$ sample volume $(25-50$ $\mu \mathrm{L})$ of buffer.

'Real-time' MAC samples were formed in situ within the AFM liquid chamber. For experiments at $37^{\circ} \mathrm{C}$ using a Bruker Dimension FastScan, the complement proteins were injected directly onto the supported lipid bilayer whilst imaging. For rapid imaging experiments performed at $30^{\circ} \mathrm{C}$ using home-built AFM instrumentation described previously ${ }^{47}$, the sample chamber was passivated with BSA $(0.1 \mathrm{mg} / \mathrm{mL}$ in incubation buffer) for $15 \mathrm{~min}$ and rinsed with $500 \mu \mathrm{L}$ buffer prior to loading the sample with the supported lipid bilayer. Complement proteins were sequentially injected though channels in the sample chamber to the imaging volume, while scanning and in the absence of wash steps, to give a final protein concentration of $80 \mathrm{nM}$ for C5b6, C7 and C8 and $1.4 \mu \mathrm{M}$ for C9.

AFM Imaging. AFM imaging was performed in fluid using a Bruker Dimension FastScan (for time-lapse imaging at $37^{\circ} \mathrm{C}$ ), a Bruker BioScope Resolve (for imaging of MAC intermediates) and a home-built instrument with rapid imaging capabilities 47,48 (for rapid imaging at $30^{\circ} \mathrm{C}$ ). Imaging was generally performed in off-resonance tapping/fast force-feedback imaging (Bruker's PeakForce Tapping) mode where force-distance curves were recorded at either 8 or $32 \mathrm{kHz}$, with amplitudes of $10-20 \mathrm{~nm}$. With these frequencies, images could be collected at 5-100 s/frame. Tapping mode of MAC intermediates was performed while scanning bidirectionally (i.e. turn around at the end of each imaging line; no 'retrace')

Rapid PeakForce Tapping (with the $\mathrm{z}$ scanner driving at $32 \mathrm{kHz}$ ) was performed with largely custom built hardware as described in detail elsewhere ${ }^{47}$. Importantly, the AFM head has a sufficiently small laser spot to accommodate miniaturised cantilevers, and the high-speed scanner is flexure-based with a $1.8 \mu \mathrm{m} \times 1.8 \mu \mathrm{m} \times 2$ $\mu \mathrm{m}$ range and $\sim 100 \mathrm{kHz} z$ bandwidth. Fast force-distance based imaging modes were implemented by sinusoidally modulating the tip-sample distance at a high rate between 16 and $32 \mathrm{kHz}$ and recording the resulting deflection signal with a significantly higher sampling rate $(512 \mathrm{kHz})$. The resulting periodic hydrodynamic background was recorded slightly above the surface and subtracted from the deflection in real time. The resulting interaction was a sinusoidal force-distance curve where the maximum force was used for feedback. For practical purposes, highest-quality data were recorded at $30^{\circ} \mathrm{C}$; and next compared with results obtained at the physiological $37^{\circ} \mathrm{C}$ (see main text).

Commercial FastScan-D cantilevers (Bruker) were used for all experiments, except for images shown in Fig. 2, which used pre-release Fast Tapping probes (Bruker; resonance frequency $140 \mathrm{kHz}$, spring constant $0.3 \mathrm{~N} \mathrm{~m}^{-1}$ ). FastScan-D cantilevers have a specified spring constant of $0.25 \mathrm{~N} \mathrm{~m}^{-1}$ with a resonance frequency of $110 \mathrm{kHz}$ in liquid; this exceeds our ramping frequency by at least a factor of 3, sufficient to avoid coupling between the ramping frequency and the cantilever resonance. Cantilevers were rinsed in isopropanol:ethanol (1:1) and plasma cleaned in air prior to use.

AFM data processing. Image analysis was performed using Nanoscope Analysis version 1.80 (Bruker) ${ }^{27}$. Briefly, images were plane levelled and line-by-line flattened with the lipid bilayer as a reference. A Gaussian filter with a full-width halfmaximum of 2 pixels (corresponding to $4 \mathrm{~nm}$ ) was used to smooth out high frequency noise where necessary.

Tracking the evolution of a growing pore, and pore counting, was performed as follows, using MATLAB (MathWorks), and the scripts described were used with Bruker's MATLAB toolbox: NSMatlabUtilites.

AFM Movie sequences were loaded into MATLAB, and a 1st order plane background subtraction was applied to each image. A template pore was userselected from the final image in the sequence. This was used as the template in a $2 \mathrm{D}$ cross-correlation analysis, applied to each image in the sequence. If features correlated with the template over a given, normalized threshold value (set as 0.6 here; user-adjusted to optimize recognition), such features were identified as MAC pores. The number of features found in each frame was defined as the pore count. Next, using the coordinates already obtained from the $2 \mathrm{D}$ cross-correlation analysis, the coordinates of the appearance and growth of unique pores were tracked, and their coordinates stored into a new array (track). This used two further parameters: (i) A maximum linking distance (typically set at $\sim 30$ pixels), which defines a pore as being the same unique pore as that detected in the previous frame, only if the coordinates of the feature were within the maximum linking distance. (ii) A maximum gap closing (in frames), which defines the number of frames in which a feature (that is within the maximum linking distance) cannot be found and yet is still defined as belonging to the same track (this reduces the risk of artefacts due to image noise). The pore count and coordinates for individual tracks were then saved into a data structure.

Next, using the track coordinates, new image sequences for each pore were cropped to within a radial distance of $25 \mathrm{~nm}$ from the centre of the feature, and including some extra frames recorded before the first appearance of a given pore. For each cropped image sequence, the average height of each frame in the sequence was calculated and saved into a new array. This analysis was repeated for several data sets, and the data saved into new data structures. Finally, the cropped image sequences and average height arrays from several experiments were concatenated. Each track was inspected by the user and any false positives (caused by having too small a threshold value for the $2 \mathrm{D}$ cross-correlation analysis) were removed. A 
Savitzky-Golay filter was applied to each remaining average height array to reduce the effect of image noise. A sigmoid function of the form

$$
f(t)=A \times \tanh \left(\frac{\left(t-t_{0}\right)}{\tau}\right)+B
$$

was fitted to each filtered, average height array. $A, B$, $t_{0}$, and $\tau$ are fitting parameters, and $3^{\star} \tau$ (which corresponds to $\sim 90 \%$ of the transition; see Supplementary Fig. 12) was defined as the width of the transition (and hence reaction time). If, from the fitting, $t_{0}$ was negative or $3^{*} \tau$ was longer than the Movie sequence, this was considered a poor fit and the data was removed. For the image sequence tracking a single MAC pore in Fig. 4, a $1.5 \mathrm{~nm}$ Gaussian filter was applied.

Pore cross-sectional measurements. Image processing was carried out in Gwyddion $^{49}$. Images were plane levelled and line-by-line flattened with the lipid bilayer as a reference. A Gaussian filter with a full-width half-maximum of 2 pixels was used to smooth out high frequency noise. Following this, a cross-section was then taken diagonally across a single MAC pore (shown in Fig. 1.) and exported. Data was plotted in Origin.

A MAC pore of structure EM- $3134^{12}$ was imported into Chimera ${ }^{50}$. Volume filtering was performed with $\sigma=14$ pixels to obtain a structure with resolution comparable to that of the AFM data. Given a resolution of $8.5 \AA$ for EM-3134, this corresponds to a physical width (of the filter) of $11.9 \mathrm{~nm}$. The volume viewer tool was optimized to ensure the full surface as shown (level 0.002). The model was then coloured by height with a black to white gradient (low to high) of -100 to $+200 \AA$ in steps of $75 \AA$. A top view image was then exported into Gwyddion ${ }^{49}$ for crosssectional analysis. A cross-sectional measurement was taken horizontally across the pore diameter against the approximate height of a surrounding membrane $(50 \AA)$, including the stalk, and exported. Data was plotted in Origin.

QCM-D measurements. Quartz crystal microbalance with dissipation monitoring (QCM-D) allows semi-quantification of mass deposition to a quartz crystal sensor. Binding assays were performed by flowing complement proteins over a lipid bilayer supported by the silicon oxide coated QCM-D sensor. Biomolecules interacting with the sensor interface give rise to a change in resonance frequency $(\Delta f)$ and energy dissipation $(\Delta D)$ of the quartz sensor. Briefly, a decrease in resonance frequency is proportional to an increase in surface-bound mass (where this includes hydrodynamically coupled solvent in addition to the biomolecules binding), whilst an increase in dissipation qualitatively correlates with an increase in the 'softness' of the film ${ }^{33}$. QCM-D measurements were performed in flow mode at a flow rate of $10 \mu \mathrm{L} / \mathrm{min}$ using a Q-Sense E4 system equipped with four Q-Sense Flow Modules (Biolin Scientific, Vastra Frolunda, Sweden) with a working temperature of $20^{\circ} \mathrm{C}$. Silicon oxide coated QCM-D sensors (QSX 303, Biolin Scientific) were used as substrates for supported lipid bilayers. Before injection, C5b6, C7, C8 and $\mathrm{C} 9$ were diluted to concentrations of $10,5,5$ and $50 \mu \mathrm{g} / \mathrm{mL}(35,54,33$ and 704 $\mathrm{nM}$ ) respectively in incubation buffer (roughly equivalent to half those use in AFM experiments). Overtones $j=3,5,7,9,11$, and 13 were recorded in addition to the fundamental resonance frequency $(4.95 \mathrm{MHz})$. Changes in dissipation $(\Delta \mathrm{D})$ and normalised frequency, $\Delta f=\Delta f_{j} / j$, for $j=5$ are presented here; all other overtones provided equivalent information.

Vesicle lysis assays. E. coli lipid extract was suspended at $7.5 \mathrm{mg} / \mathrm{mL}$ in calcein solution (50 mM calcein, $150 \mathrm{mM} \mathrm{NaCl}, 20 \mathrm{mM}$ Hepes pH 7.4), freeze-thawed 6 times (liquid nitrogen $-65^{\circ} \mathrm{C}$ ) and extruded through a $100 \mathrm{~nm}$ polycarbonate membrane (Whatman) to form unilamellar calcein-encapsulated liposomes. Nonencapsulated calcein was removed through liposomes purification on a gravity-flow Sephadex-G50 (GE Healthcare) column (500 mM Sucrose, $150 \mathrm{mM} \mathrm{NaCl}, 20 \mathrm{mM}$ Hepes $\mathrm{pH}$ 7.4) and liposomes were used immediately. MAC lysis assays of liposomes were performed by sequential addition of $\mathrm{C} 5 \mathrm{~b} 6\left(5 \mathrm{~min}, 37^{\circ} \mathrm{C}\right), \mathrm{C} 7(5 \mathrm{~min}$, $\left.37^{\circ} \mathrm{C}\right), \mathrm{C} 8$ and $\mathrm{C} 9$ at a mass ratio of 1:1:1:1. In control conditions, identical volumes of protein buffer $(120 \mathrm{mM} \mathrm{NaCl}, 10 \mathrm{mM}$ Hepes $\mathrm{pH}$ 7.4) were added instead of protein. Self-quenched encapsulated calcein was un-quenched through its release in the extra-liposomal solution following MAC lesions. Fluorescence was recorded immediately following $\mathrm{C} 9$ addition and every minute for $60 \mathrm{~min}$ on a SpectraMax M2 fluorometer (dual monochromator, ex: $490 \mathrm{~nm}$, em: $520 \mathrm{~nm}$ ) (Molecular Devices). Background fluorescence was measured from calceinencapsulated liposomes in the absence of protein and subtracted from the data. To determine the percentage of lysis, the fluorescence was then normalized to the maximal lysis fluorescence estimated after a freeze-thaw cycle of liposomes incubated in $0.25 \%$ sodium dodecyl sulphate (SDS). Fluorescence measures of lysis and controls were always performed on the same batch of liposomes and in three independent replicates.

Negative-stain EM. Supported lipid bilayers were formed as described for equivalent supported lipid bilayers as used in AFM experiments, using $8 \mathrm{~nm}$ thick $\mathrm{PELCO}^{\circledR}$ silicon dioxide support films for transmission electron microscopy grids (Agar) as the support instead of mica. Briefly, the glow-discharged grids were incubated with an SUV suspension in calcium containing incubation buffer $(20$ $\mathrm{mM}$ HEPES, $120 \mathrm{mM} \mathrm{NaCl}, 10 \mathrm{mM} \mathrm{CaCl}_{2}$ ), rinsed and incubated with complement proteins as described above. Importantly, this allowed us to remove all soluble protein and excess lipid from incubation buffer prior to staining. Samples grids were rinsed with $500 \mu \mathrm{l}$ incubation buffer, taking care that they remained hydrated throughout, and subsequently stained with $2 \% \mathrm{wt} / \mathrm{wt}$ uranyl acetate. The sample was incubated with uranyl acetate for $60 \mathrm{~s}$ and carefully blotted dry, ensuring that the strain was quickly removed to avoid crystallisation of excess uranyl acetate at the surface. Samples were imaged on a Tecnai T12 thermionic filament microscope (Thermo Fisher Scientific) at $120 \mathrm{kV}$. Images were taken with a defocus of $0.5-1 \mu \mathrm{m}$ on a Gatan $4 \mathrm{k} \times 4 \mathrm{k}$ CCD camera, giving a final pixel size of $1.64 \AA$.

Fluorescence recovery after photobleaching. E. coli lipid extract was doped with $0.5 \mathrm{~mol} \%$ Topfluor PC by mixing the respective lipids in chloroform and preparing SUV $^{27}$. A thin mica slide was suspended over a $10 \mathrm{~mm}$ glass window in a glass bottomed cell culture plate. Deposition of fluorescently doped SUVs was achieved as described above, taking care to ensure that the mica support remained hydrated at all time. Lipid bilayers were transferred to a FV1200 confocal microscope equipped with a $100 \times 1.40$ oil immersion objective (both Olympus) and a TC-324B automatic temperature controller (Warner Instruments) set to $37^{\circ} \mathrm{C}$. The microscope was further set to a FV10-LD473 $473 \mathrm{~nm}$ and $15 \mathrm{~mW}$ laser diode powered by a FV10MCPSU power supply, and the Alexa 488 excitation filter and BA490-590 emission filter sets (all Olympus). The fluorescent bilayers were imaged at $2.5 \%$ laser output power across $49.8 \mu \mathrm{m}$ wide areas and an acquisition speed of $1.64 \mathrm{~s}$ per image. FRAP was performed on a circular area of $16.4 \mu \mathrm{m}$ diameter at the centre of an image for $2 \mathrm{~s}$ at a laser output of $80 \%$. Analysis of the FRAP data was performed according to the Soumpasis model for diffusion limited recovery ${ }^{24,42}$.

Kinetic analysis. Given that the initiation and prolongation of C9 binding to C5b-8 occur at such different time scales ( $\tau_{\text {init }}$ and $\tau_{\text {olig }}$ or $\tau_{+}$, respectively) we approximate the reaction kinetics by considering the separate reactions:

$$
\mathrm{C} 5 \mathrm{~b}-8+\mathrm{C} 9 \stackrel{k_{\text {init }}}{\rightarrow} \mathrm{C} 5 \mathrm{~b}-8(\mathrm{C} 9)_{1}
$$

and

$$
\text { C5b-8 }(\mathrm{C} 9)_{n}+\mathrm{C} 9 \stackrel{k_{+}}{\rightarrow} \text { C5b-8 }(\mathrm{C} 9)_{n+1}(1 \leq n<18),
$$

while assuming an excess (and therefore approximately constant) amount of $\mathrm{C} 9$ in solution (see main text).

The kinetics of the initiation reaction then follows from the differential equation

$$
\mathrm{d}\left[\mathrm{C} 5 \mathrm{~b}-8(\mathrm{C} 9)_{\mathrm{n}}\right] / \mathrm{d} t=k_{\text {init }}[\mathrm{C} 5 \mathrm{~b}-8][\mathrm{C} 9],
$$

where square brackets denote concentrations. For a single C5b- 8 complex, we define the initiation probability:

$$
p_{\text {init }} \equiv\left[\mathrm{C} 5 \mathrm{~b}-8(\mathrm{C} 9)_{1}\right] /\left([\mathrm{C} 5 \mathrm{~b}-8]+\left[\mathrm{C} 5 \mathrm{~b}-8(\mathrm{C} 9)_{1}\right]\right) .
$$

This leads to a solution of the form:

$$
p_{\text {init }}=1-\exp \left(-k_{\text {init }}[\mathrm{C} 9] t\right) \text {. }
$$

such that $k_{\text {init }} \approx \tau_{\text {init }}{ }^{-1}[\mathrm{C} 9]^{-1}$, with $\tau_{\text {init }}$ determined as illustrated in Fig. $3 \mathrm{~b}$. Defining

$$
p_{n} \equiv\left[\mathrm{C} 5 \mathrm{~b}-8(\mathrm{C} 9)_{n}\right] / \sum_{n=1}^{18}\left[\mathrm{C} 5 \mathrm{~b}-8(\mathrm{C} 9)_{n}\right],
$$

we can describe the subsequent $\mathrm{C} 9$ oligomerization via the coupled differential equations

$$
\mathrm{d} p_{1} / \mathrm{d} t=-k_{+}[\mathrm{C} 9] p_{1}
$$

and

$$
\mathrm{d} p_{n} / \mathrm{d} t=k_{+}[\mathrm{C} 9] p_{n-1}-k_{+}[\mathrm{C} 9] p_{n}
$$

for $2 \leq n<18$, and $\mathrm{d} p_{18} / \mathrm{d} t=k_{+}[\mathrm{C} 9] p_{17}$

The average oligomerization rate is given in number of added $\mathrm{C} 9$ molecules per unit of time, as

$$
\begin{array}{r}
\mathrm{d}\langle n\rangle / \mathrm{d} t=\sum_{n=1}^{18} n \mathrm{~d} p_{n} / \mathrm{d} t=k_{+}[\mathrm{C} 9]\left(\sum_{n=2}^{18} n p_{n-1}-\sum_{n=1}^{17} n p_{n}\right) \\
=k_{+}[\mathrm{C} 9]\left(\sum_{n=1}^{17}(n+1) p_{n}-\sum_{n=1}^{17} n p_{n}\right)=k_{+}[\mathrm{C} 9] \sum_{n=1}^{17} p_{n} .
\end{array}
$$

Taking into account that $\sum_{n=1}^{18} p_{n}=1$ and assuming that $p_{18}$ is significantly smaller than 1 (i.e., ignoring the oligomerization of the last few C9s), we find a constant oligomerization rate

$$
\mathrm{d}\langle n\rangle / \mathrm{d} t \approx k_{+}[\mathrm{C} 9],
$$

such that

$$
k_{+} \approx \tau_{+}^{-1}[\mathrm{C} 9]^{-1},
$$

with $\tau_{+}$determined as in Fig. 4 .

Reporting summary. Further information on research design is available in the Nature Research Reporting Summary linked to this article. 


\section{Data availability}

Data supporting the findings of this manuscript are available from the corresponding authors upon reasonable request. A reporting summary for this Article is available as a Supplementary Information file. The source data underlying Figs. 2b, 3b, 4b,c and Supplementary Figs. $2 \mathrm{c}, 3 \mathrm{c}-\mathrm{e}, 7 \mathrm{a}-\mathrm{b}, 8 \mathrm{a}-\mathrm{b}$ and $9 \mathrm{~b}$ are provided as a Source Data file.

\section{Code availability}

Analysis code to extract kinetic parameters from rapid AFM imaging data is available on github. An example AFM data set is provided as a MATLAB data structure to enable assessment of the analysis code.

Received: 27 November 2018 Accepted: 12 April 2019

Published online: 06 May 2019

\section{References}

1. Podack, E. R. \& Munson, G. P. Killing of microbes and cancer by the immune system with three mammalian pore-forming killer proteins. Front. Immunol. 7, 263 (2016)

2. Dal Peraro, M. \& van der Goot, F. G. Pore-forming toxins: ancient, but never really out of fashion. Nat. Rev. Microbiol. 14, 77-92 (2016).

3. Lukoyanova, N., Hoogenboom, B. W. \& Saibil, H. R. The membrane attack complex, perforin and cholesterol-dependent cytolysin superfamily of poreforming proteins. J. Cell Sci. 129, 2125-2133 (2016).

4. Kaufmann, S. H. E. Immunology's foundation: the 100-year anniversary of the Nobel Prize to Paul Ehrlich and Elie Metchnikoff. Nat. Immunol. 9, 705-712 (2008).

5. Morgan, B. P. \& Harris, C. L. Complement, a target for therapy in inflammatory and degenerative diseases. Nat. Rev. Drug Disco. 14, 857-877 (2015).

6. Harder, M. J. et al. Incomplete inhibition by eculizumab: mechanistic evidence for residual C5 activity during strong complement activation. Blood 129, 970-980 (2017).

7. Lindorfer, M. A. et al. Real-time analysis of the detailed sequence of cellular events in mAb-mediated complement-dependent cytotoxicity of B-cell lines and of chronic lymphocytic leukemia B-cells. Mol. Immunol. 70, 13-23 (2016).

8. Taylor, R. P. \& Lindorfer, M. A. Cytotoxic mechanisms of immunotherapy: Harnessing complement in the action of anti-tumor monoclonal antibodies. Semin. Immunol. 28, 309-316 (2016).

9. Reis, E. S., Mastellos, D. C., Ricklin, D., Mantovani, A. \& Lambris, J. D Complement in cancer: untangling an intricate relationship. Nat. Rev Immunol. 18, 5-18 (2018).

10. Heesterbeek, D. A. et al. Bacterial killing by complement requires membrane attack complex formation via surface-bound C5 convertases. EMBO J. $\mathbf{3 8}$ e99852 (2019).

11. DiScipio, R. G., Smith, C. A., Müller-Eberhard, H. J. \& Hugli, T. E. The activation of human complement component $\mathrm{C} 5$ by a fluid phase $\mathrm{C} 5$ convertase. J. Biol. Chem. 258, 10629-10636 (1983).

12. Serna, M., Giles, J. L., Morgan, B. P. \& Bubeck, D. Structural basis of complement membrane attack complex formation. Nat. Commun. 7, 10587 (2016).

13. Menny, A. et al. CryoEM reveals how the complement membrane attack complex ruptures lipid bilayers. Nat. Commun. 9, 5316 (2018).

14. Dudkina, N. V. et al. Structure of the poly-C9 component of the complement membrane attack complex. Nat. Commun. 7, 10588 (2016).

15. Spicer, B. A. et al. The first transmembrane region of complement component-9 acts as a brake on its self-assembly. Nat. Commun. 9, 3266 (2018).

16. Sharp, T. H., Koster, A. J. \& Gros, P. Heterogeneous MAC initiator and pore structures in a lipid bilayer by phase-plate cryo-electron tomography. Cell Rep. 15, 1-8 (2016)

17. Hadders, M. A. et al. Assembly and regulation of the membrane attack complex based on structures of C5b6 and sC5b9. Cell Rep. 1, 200-207 (2012).

18. Lovelace, L. L., Cooper, C. L., Sodetz, J. M. \& Lebioda, L. Structure of human C8 protein provides mechanistic insight into membrane pore formation by complement. J. Biol. Chem. 286, 17585-17592 (2011).

19. Meri, S. et al. Human protectin (CD59), an 18,000-20,000 MW complement lysis restricting factor, inhibits C5b-8 catalysed insertion of C9 into lipid bilayers. Immunology 71, 1-9 (1990).

20. Farkas, I. et al. CD59 blocks not only the insertion of C9 into MAC but inhibits ion channel formation by homologous C5b-8 as well as C5b-9. J. Physiol. 539, 537-545 (2002).
21. Kawachi-Takahashi, S., Tanaka, K., Takahashi, M., Kawashima, T. \& Shimada K. Determination of Serum C9 Level by Immunodiffusion. Int. Arch. Allerg. Immunol. 48, 161-170 (1975).

22. Oursel, D. et al. Lipid composition of membranes of Escherichia coli by liquid chromatography/tandem mass spectrometry using negative electrospray ionization. Rapid Commun. Mass Spectrom. 21, 1721-1728 (2007).

23. Israelachvili, J. N. Intermolecular and Surface Forces. (Academic Press, Burlington MA, 2011)

24. Sims, P. J. \& Wiedmer, T. Kinetics of polymerization of a fluoresceinated derivative of complement protein $\mathrm{C} 9$ by the membrane-bound complex of complement proteins C5b-8. Biochemistry 23, 3260-3267 (1984).

25. Marshall, P., Hasegawa, A., Davidson, E. A., Nussenzweig, V. \& Whitlow, M Interaction between complement proteins C5b-7 and erythrocyte membrane sialic acid. J. Exp. Med. 184, 1225-1232 (1996).

26. Silversmith, R. E. \& Nelsestuen, G. L. Interaction of complement proteins C5b-6 and C5b-7 with phospholipid vesicles: effects of phospholipid structural features. Biochemistry 25, 7717-7725 (1986)

27. Leung, C. et al. Real-time visualization of perforin nanopore assembly. Nat. Nanotechnol. 12, 467-473 (2017).

28. Leung, C. et al. Stepwise visualization of membrane pore formation by suilysin, a bacterial cholesterol-dependent cytolysin. eLife 3, e04247 (2014).

29. Stewart, S. E. et al. Analysis of perforin assembly by quartz crystal microbalance reveals a role for cholesterol and calcium-independent membrane binding. J. Biol. Chem. 290, 31101-31112 (2015).

30. Stewart, S. E. et al. Assembly of streptolysin O pores assessed by quartz crystal microbalance and atomic force microscopy provides evidence for the formation of anchored but incomplete oligomers. Biochim. Biophys. Acta 1848, 115-126 (2015).

31. Yorulmaz, S. et al. Membrane attack complex formation on a supported lipid bilayer: initial steps towards a CARPA predictor nanodevice. Eur. J. Nanomed. 7, 151-255 (2015)

32. Yorulmaz, S., Jackman, J. A., Hunziker, W. \& Cho, N.-J. Supported lipid bilayer platform to test inhibitors of the membrane attack complex: insights into biomacromolecular assembly and regulation. Biomacromolecules 16, 3594-3602 (2015)

33. Reviakine, I., Johannsmann, D. \& Richter, R. P. Hearing what you cannot see and visualizing what you hear: interpreting quartz crystal microbalance data from solvated interfaces. Anal. Chem. 83, 8838-8848 (2011).

34. Ruan, Y., Rezelj, S., Bedina Zavec, A., Anderluh, G. \& Scheuring, S. Listeriolysin $\mathrm{O}$ membrane damaging activity involves arc formation and lineaction-implication for listeria monocytogenes escape from phagocytic vacuole. PLoS Pathog. 12, e1005597-18 (2016).

35. Hotze, E. M. et al. Monomer-monomer interactions propagate structural transitions necessary for pore formation by the cholesterol-dependent cytolysins. J. Biol. Chem. 287, 24534-24543 (2012).

36. Ramachandran, R., Tweten, R. K. \& Johnson, A. E. Membrane-dependent conformational changes initiate cholesterol-dependent cytolysin oligomerization and intersubunit beta-strand alignment. Nat. Struct. Mol. Biol. 11, 697-705 (2004).

37. van Pee, K. et al. CryoEM structures of membrane pore and prepore complex reveal cytolytic mechanism of Pneumolysin. eLife 6, 10210 (2017).

38. Huang, Y., Qiao, F., Abagyan, R., Hazard, S. \& Tomlinson, S. Defining the CD59-C9 Binding Interaction. J. Biol. Chem. 281, 27398-27404 (2006).

39. Law, R. H. P. et al. The structural basis for membrane binding and pore formation by lymphocyte perforin. Nature 468, 447-451 (2010).

40. Kessel, M., Brennan, M. J., Trus, B. L., Bisher, M. E. \& Steven, A. C. Naturally crystalline porin in the outer membrane of Bordetella pertussis. J. Mol. Biol. 203, 275-278 (1988).

41. Sohlenkamp, C. \& Geiger, O. Bacterial membrane lipids: diversity in structures and pathways. FEMS Microbiol. Rev. 40, 133-159 (2016).

42. Aleshin, A. E., DiScipio, R. G., Stec, B. \& Liddington, R. C. Crystal structure of C5b-6 suggests structural basis for priming assembly of the membrane attack complex. J. Biol. Chem. 287, 19642-19652 (2012).

43. Chonn, A., Cullis, P. R. \& Devine, D. V. The role of surface charge in the activation of the classical and alternative pathways of complement by liposomes. J. Immunol. 146, 4234-4241 (1991).

44. Ninomiya, H. \& Sims, P. J. The human complement regulatory protein CD59 binds to the alpha-chain of C8 and to the 'b'domain of C9. J. Biol. Chem. 267, 13675-13680 (1992)

45. Lockert, D. H. et al. Identity of the segment of human complement C8 recognized by complement regulatory protein CD59. J. Biol. Chem. 270, 19723-19728 (1995)

46. Richter, R. P., Bérat, R. \& Brisson, A. R. Formation of solid-supported lipid bilayers: an integrated view. Langmuir 22, 3497-3505 (2006).

47. Nievergelt, A. P., Banterle, N., Andany, S. H., Gönczy, P. \& Fantner, G. E High-speed photothermal off-resonance atomic force microscopy reveals assembly routes of centriolar scaffold protein SAS-6. Nat. Nanotechnol. 1 https://doi.org/10.1038/s41565-018-0149-4 (2018). 
48. Nievergelt, A. P., Andany, S. H., Adams, J. D., Hannebelle, M. T. \& Fantner, G. E. Components for high-speed atomic force microscopy optimized for low phase-lag. 731-736 https://doi.org/10.1109/AIM.2017.8014104 (IEEE, 2017).

49. Nečas, D. \& Klapetek, P. Gwyddion: an open-source software for SPM data analysis. Open Phys. 10, 99-8 (2011).

50. Pettersen, E. F. et al. UCSF Chimera-a visualization system for exploratory research and analysis. J. Comput Chem. 25, 1605-1612 (2004).

\section{Acknowledgements}

We thank Richard Thorogate for technical support, Natalya Lukoyanova and Shu Chen for training and advice on EM, and Andrea Slade and James Shaw (Bruker) for advice and assistance with and access to their AFM equipment. This work has been funded by the UK BBSRC and MRC project grants (BB/N015487/1 and MR/R000328/1, to B.W.H.); UK EPSRC and MRC fellowships (EP/M507970/1 to E.S.P.; EP/M506448/1 and MR/ R024871/1 to A.L.B.P.); UK BBSRC studentships (BB/J014567/1 for G.J.S. and BB/ M503113/1 for A.R.Y.); and UK EPSRC investment in AFM equipment (EP/M028100/1). A.M. and D.B. are supported by a CRUK Career Establishment Award (C26409/A16099) to D.B. G.E.F and A.P.N acknowledge funding from the European Union FP7/ 2007-2013/ERC under Grant Agreement No. 307338-NaMic and the European Union H2020 Framework Programme for Research \& Innovation (2014-2020); ERC-2017-CoG; InCell; Project number 773091.

\section{Author contributions}

E.S.P. conceived the study, carried out AFM, QCM-D, EM and FRAP experiments, analysed data, led the research and wrote the manuscript. G.J.S. developed and performed tracking analysis of AFM data. A.L.B.P. contributed protocols for, advised on and assisted with AFM experiments, analysed data and wrote the manuscript. A.W.H. performed EM and FRAP experiments. A.P.N. provided home-built instrumentation and assisted with AFM experiments. A.M. performed EM and lysis experiments. A.R.Y. assisted with AFM experiments. A.R. assisted with QCM-D experiments. R.P.R. conceived and advised on QCM-D experiments, and contributed to data analysis and interpretation. G.E.F. provided homebuilt instrumentation and advised on AFM experiments. D.B. advised on EM and lysis experiments and on structural aspects of the MAC, and wrote the manuscript. B.W.H. conceived the study, developed the tracking analysis of the AFM data, analysed data, led the research and wrote the manuscript. All authors reviewed and commented on the manuscript and its intellectual content.

\section{Additional information}

Supplementary Information accompanies this paper at https://doi.org/10.1038/s41467019-10058-7.

Competing interests: The authors declare no competing interests.

Reprints and permission information is available online at http://npg.nature.com/ reprintsandpermissions/

Journal peer review information: Nature Communications thanks George Heath and the other anonymous reviewer(s) for their contribution to the peer review of this work Peer reviewer reports are available.

Publisher's note: Springer Nature remains neutral with regard to jurisdictional claims in published maps and institutional affiliations.

(c) (i) Open Access This article is licensed under a Creative Commons Attribution 4.0 International License, which permits use, sharing, adaptation, distribution and reproduction in any medium or format, as long as you give appropriate credit to the original author(s) and the source, provide a link to the Creative Commons license, and indicate if changes were made. The images or other third party material in this article are included in the article's Creative Commons license, unless indicated otherwise in a credit line to the material. If material is not included in the article's Creative Commons license and your intended use is not permitted by statutory regulation or exceeds the permitted use, you will need to obtain permission directly from the copyright holder. To view a copy of this license, visit http://creativecommons.org/ licenses/by/4.0/.

(C) The Author(s) 2019 